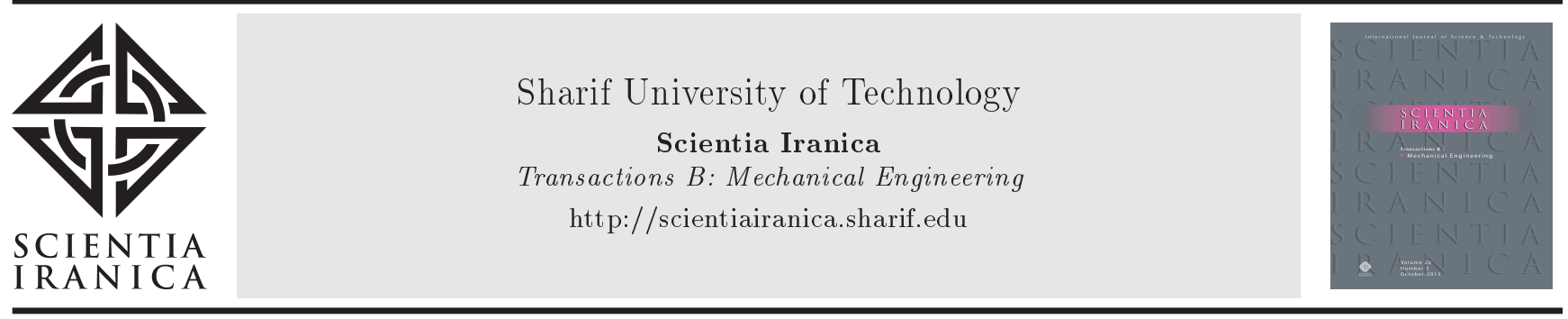

Research Note

\title{
A parametric study on the mechanical role of fibrillar rotations in an articular cartilage finite element model
}

\author{
S.S. Sajjadinia ${ }^{a}$ and M. Haghpanahi ${ }^{b, *}$ \\ a. Faculty of Computer Science, Free University of Bozen-Bolzano, 39100 Bozen-Bolzano, Italy. \\ b. School of Mechanical Engineering, Iran University of Science and Technology, Tehran 16846, Iran. \\ Received 15 September 2018; received in revised form 14 January 2020; accepted 11 May 2020
}

\section{KEYWORDS \\ Biomechanics; \\ Articular cartilage; \\ Fibrillar rotation; \\ UMAT; \\ Parametric finite \\ element analysis.}

\begin{abstract}
Collagen network is one of Articular Cartilage (AC) vital components that contributes to the depth-dependent and anisotropic responses of the tissue. Since it is computationally expensive to simulate all the structural details of the AC network, they are typically simplified in numerical analysis. In particular, the so-called arcade-like structure, which has been widely used in previous complex simulations, does not capture the rotations of fibrillar bundles. In this study, the role of such possible rotations in the AC mechanical response was investigated by a set of advanced, biphasic, and parametric Finite Element (FE) simulations of indentation tests. The obtained results indicated the influence of Fibrillar Rotations (FRs) on the mechanical response by increasing the fibrillar stress while regionally affecting the stress in the upper layers of the AC tissue. On the contrary, FR did not significantly alter the tissue elasticity and consequently, might be safely ignored in pure contact mechanical problems. It can be concluded that the excessive FR might regionally increase the stress, which could have a degenerative effect on the collagen constituent; therefore, it should not be neglected in the related future studies, in which the upper AC layers would resist high permanent shear strains.
\end{abstract}

(C) 2021 Sharif University of Technology. All rights reserved.

\section{Introduction}

Articular Cartilage (AC) is an essential tissue of human knee joints consisting of several discrete components that play a vital role in knee functions by allowing the nearly frictionless motion between the contacting surfaces [1,2]. Moreover, the AC tissue is reinforced by a type-II collagen network, stimulating an anisotropic and heterogeneous response of the tissue [3]. Collagens are protein molecules that form a fibrillar network of

*. Corresponding author. Tel.: +9821 77240540-50 E-mail addresses: seyedshayan.sajjadinia@stud-inf.unibz.it (S.S. Sajjadinia); mhaghpanahi@iust.ac.ir (M. Haghpanahi)

doi: $10.24200 /$ sci.2020.51785.2362
$\mathrm{AC}$ and improve cartilage elasticity by augmenting the tensile stiffness [1]. Accordingly, accurate modeling of the collagen network is an essential step in most of the AC simulations.

For decades, a variety of constitutive equations have been developed to address the AC fibrillar response [4-8]. Although those models could thoroughly simulate the fibrillar part of AC, they are numerically, too expensive to be implemented [9]. As a result, most of the practical 3D studies on Finite Elements (FEs) were conducted through simplification of the geometrical and material characteristics of $\mathrm{AC}$ components. In particular, some of these previous studies [10-14] assumed that primary fibrils were located on planar surfaces, perpendicular to the AC surface. Intersection of those surfaces and the AC surface could form a de- 
tectable pattern of split lines (Figure 1). However, this assumption can be violated, considering that the $\mathrm{AC}$ surface would experience rotational deformation under permanent shear strains which can rotate the split lines as well. For instance, such permanent rotations might occur in knees with rotational deformity [15]. Since the split lines were formed by the primary fibrils on the AC surface, their rotation should also rotate the corresponding AC fibrils so that they would not lie on a single plane.

Although the significance of fibrillar directions in their planar surfaces has been previously examined [3], [16-19], to the best of the authors' knowledge, there is no published study on the biomechanical influence of Fibrillar Rotations (FR), caused by the split line rotation (Figure 1). To this end, in the present study, an advanced parametric FE simulation was performed through a general and anisotropic poroelastic constitutive model of the knee AC cuboid sample to compare the tissue-scale response of cartilage with and without FR. The main objective of this study was to simulate the effects of FR on the elasticity and biphasic response of AC different zones, i.e., Superficial Zone (SZ), Middle Zone (MZ), and Deep Zone (DZ), using a combination of indentation tests. In this respect, we hypothesized that FR might have considerable effects on the AC mechanical response.

\section{Materials and methods}

\subsection{Constitutive model}

Simulation of this study requires implementation of a poroelastic model of AC [20], in which the total Cauchy stress $\boldsymbol{\sigma}^{T O T}$ is achieved by the summation of the contributions of hydrostatic fluid (pore) pressure $p$ and solid stresses, including the non-fibrillar Cauchy stress $\sigma^{M A T}$ in solid AC parts and tensile Cauchy stress $\boldsymbol{\sigma}^{C O L}$ in the fibrillar network. Therefore, the AC total stress is defined by [21]:

$$
\boldsymbol{\sigma}^{T O T}=\boldsymbol{\sigma}^{C O L}+\boldsymbol{\sigma}^{M A T}-p \mathbf{I}
$$

where $\mathbf{I}$ is the unit tensor. In this study, the equation for the fibrillar collagen stress was adapted from the model proposed by Wilson et al. [5,12], as follows:

$$
\boldsymbol{\sigma}^{T O T}=\sum\left(\sigma^{F} \mathrm{n} \otimes \mathrm{n}\right)
$$

where $\mathbf{n}$ is a unit vector in the current direction of each fibril, $\otimes$ is the dyadic product, and $\boldsymbol{\sigma}^{F}$ is the corresponding fibrillar tensile stress which is given by:

$$
\boldsymbol{\sigma}^{F}=\frac{\lambda \phi^{0} \rho E_{f}}{J} \quad \text { if } \quad \lambda>1,
$$

where $J$ is the determinant of the deformation gradient tensor, $\lambda$ is the elongation of each fibril, $\rho$ is the volume fraction of the collagen fibrils in each direction with respect to the total solid matrix volume, $E_{f}$ is a positive material coefficient, and $\phi^{0}$ is the initial solid volume fraction.

Moreover, for the non-fibrillar part, an improved version of the compressible Neo-Hookean model was utilized, proposed by Wilson et al. [12] in the following equation:

$$
\begin{aligned}
\boldsymbol{\sigma}^{M A T} & =\frac{\phi^{0}(1-\rho) G_{m}}{6 J}\left[6\left(\mathbf{B}-J^{2} / 3 \mathbf{I}\right)\right. \\
& \left.-\ln J\left(\frac{3 J \phi^{0} \ln J}{\left(J-\phi^{0}\right)^{2}}-1-3 \frac{J+\phi^{0}}{J-\phi^{0}}\right) \mathbf{I}\right],
\end{aligned}
$$

where $\mathbf{B}$ is the left Cauchy-Green deformation tensor and $G_{m}$ is the shear modulus.

\subsection{FE implementation}

In this study, collagen fibers in each location consist of two large primary fibrils parallel to the arcade-like orientation $[5,22]$ and seven smaller secondary fibrils which are isotopically organized. The primary fibrils

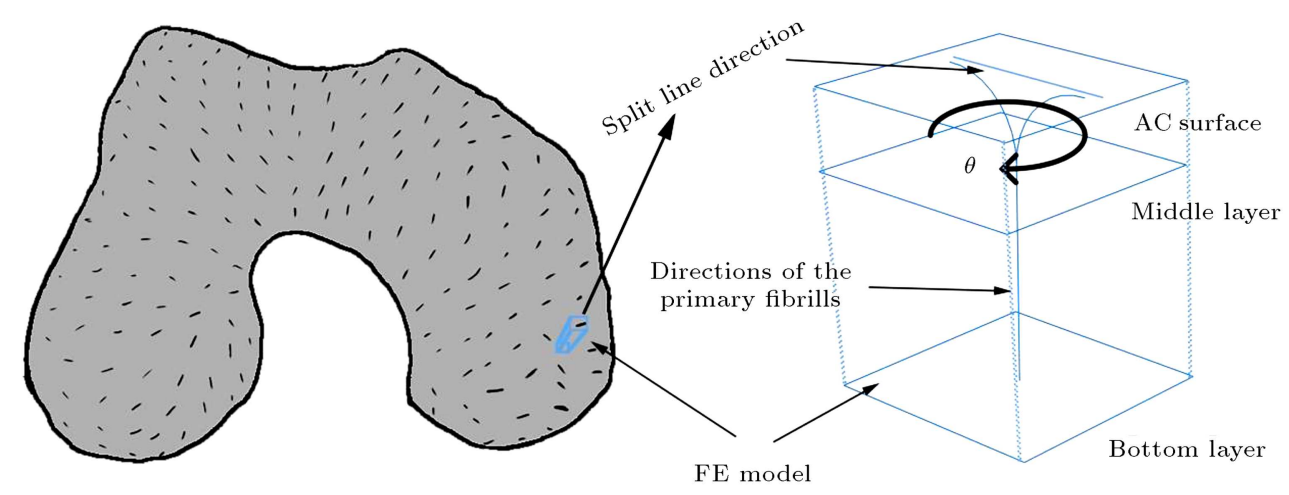

Figure 1. Schematic joint scale image of split line patterns (left) and their dependencies on the directions of the primary fibrils in the tissue-scale and cuboid FE model of AC (right) with consideration of the split line rotation of $\theta$, which would take the Fibrillar Rotation (FR) from AC surface to the middle layer (re-drawn from [5,30]); AC: Articular Cartilage; FE: Finite Element. 
extend perpendicularly from the bottom layer and bend in the opposite directions in the MZ, above the middle layer, to become parallel to the AC surface. The influence of FR was implemented by rotating the primary fibrils through an angle of $\theta$ around an axis perpendicular to the AC surface, as shown in Figure 1. It was assumed that $\mathrm{FR}$ values were linearly dependent on the normalized depth of $\mathrm{AC}(Z)$.

The FE model of the biphasic constitutive equations of $\mathrm{AC}$ was solved using the soils consolidation theory in ABAQUS (v6.14, Simulia). First, the initial values for the depth-dependent material parameters in the solid AC parts, i.e., the solid volume fraction, direction, and volume fraction of the collagen fibrils, were calculated in each integration point by a userdefined solution-dependent state variables initialization (SDVINI) subroutine. Then, the calculated parameters were taken to the user-defined material (UMAT) subroutine, which is necessary for the implementation of complex AC solid constituents [5]. In doing so, the fibrillar components and their rotations were considered in the constitutive equations implemented by the Fortran UMAT subroutine. It is suggested that readers refer to the previous studies [21] for a more detailed explanation of the implementation of the AC model.

In order to highlight the effects of FR, some parametric studies were carried out by changing the range of FR (in degrees) via a PYTHON script in Abaqus (Figure 2) based on the following equation:

$$
\theta=4.5 M, \quad \text { for } \quad M=\{1,2,3, \ldots, 40\} .
$$

Ideally, the $\mathrm{AC}$ could have been modeled by $3 \mathrm{D}$ elements, but these element types are computationally expensive [23]. Therefore, for general models [10,17,24], 2D mesh are preferred. Accordingly, indentation tests were simulated by a plane strain mesh consisting of 630 linear quadrilateral elements of type CPE4P. This mesh is associated with a cuboid plug of $\mathrm{AC}$ with the thickness of $1 \mathrm{~mm}$ and length of $4 \mathrm{~mm}$, which is contacted by an indenter with a radius of $0.5 \mathrm{~mm}$. While the nodal displacements at the bottom layer were confined, the rigid permeable indenter was placed on top. In addition, the zero pore pressure was set at the bottom and top surfaces (i.e., the fluid was allowed to flow freely on these surfaces). The indenter was pushed down with $10 \%$ strains at two different ramp rates of 0.02 and $2 \mathrm{Sec}^{-1}$, respectively, while the $\mathrm{AC}$ reaction force and stresses in each $\mathrm{AC}$ zone and constituent were recorded.

Herein, $Z$ is assumed as the normal depth of the tissue. Other material parameters were adapted from some previous research studies $[9,12,25-27]$ and set as follows:

$$
\begin{aligned}
& \phi^{0}=0.1 Z+0.1, \quad \rho=1.4 Z^{2}-1.1 Z+0.59, \\
& G_{m}=0.65 \mathrm{MPa}, \quad E_{f}=5.80 \mathrm{MPa} .
\end{aligned}
$$

Also, the permeability parameter was assumed 0.001 $\mathrm{mm}^{4} /$ N.Sec.

\section{Results and discussion}

This study employed a parametric FE simulation in order to determine the role of $\mathrm{FR}$ in the $\mathrm{AC}$ biomechanical response. The rotation was presumed to occur in the area above the middle surface and to be linearly dependent on the normalized depth (Figure 1). The numerical procedure was implemented by a combination of different tools: ABAQUS, PYTHON scripting, Fortran UMAT, and SDVINI subroutines (Figure 2). The results highlighted the role of FR in elasticity and stress distributions of AC, which could be used to estimate the significance of FR mechanical response at different scales.

In order to simulate the effect of FR, a set of indentation tests was implemented with the maximum strain of $10 \%$ and two different strain rates $(0.002$ and $2 \mathrm{Sec}^{-1}$ ) for each rotation, which was assumed to be in the range of $0-2 \pi \operatorname{Rad}$. The simulation was carried out using a poroelastic model with a focus on the fibrillar directions.

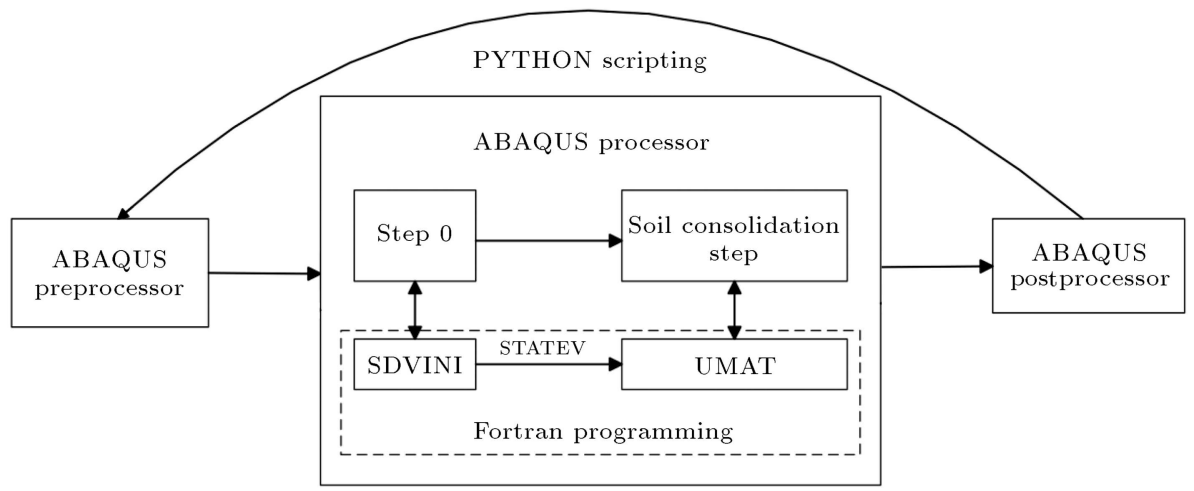

Figure 2. Methodology of Finite Element (FE) simulation. 
In each test, the axial stress in the areas below the indenter and between each zone was recorded (Figure 3). As expected, the stress magnitudes in the DZ were not affected by the rotations, since this zone was devoid of any FR. In contrast, the stress magnitudes in the SZ rose, being notably more observable with the FR ranging from 1.5 to 2.5 Rad. Despite the excessive decrease of FR impacts in the MZ, this result could still support the hypothesis. Figure 3 presents a variation in the effects of FR depending on the loading rates, but a similar tendency in both strain rates is discernable; for instance, FR can affect the stresses of AC components disregarding the loading rate.

Furthermore, the recorded reaction forces considering both of the strain rates of 0.002 and $2 \mathrm{Sec}^{-1}$ were calculated for a range of FRs (Figure 4), showing slight increases in the forces in both of the tests. In other words, the FR could slightly increase the AC elasticity. As a result, the FR modeled in this study might not affect the results beyond the tissue scale and could be overlooked in the knee joint scale studies. This finding agrees with previous works, suggesting that fibrillar organization could generally affect the tissuescale stress distributions $[9,28]$.

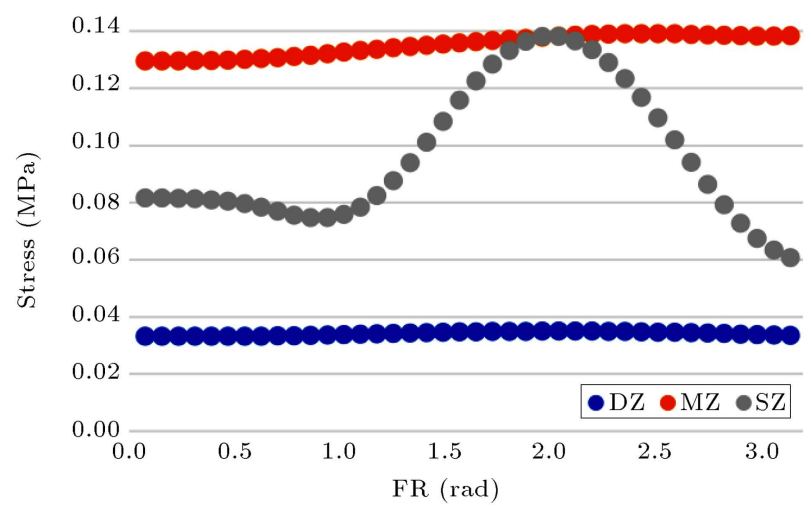

(a)

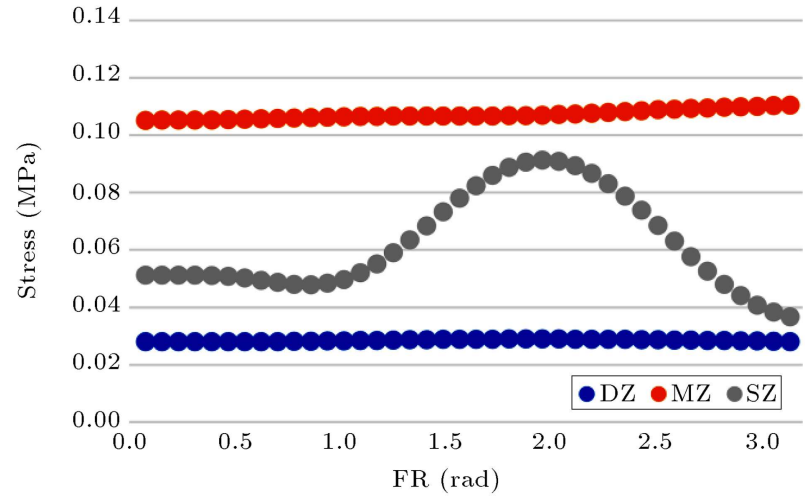

(b)

Figure 3. Mises stress in each Articular Cartilage (AC) zone for all FRs. The results are derived during the strain rates of (a) 0.02 and (b) $2 \mathrm{Sec}^{-1}$; SZ: Superficial Zone; MZ: Middle Zone; DZ: Deep Zone; FR: Fibrillar Rotation.

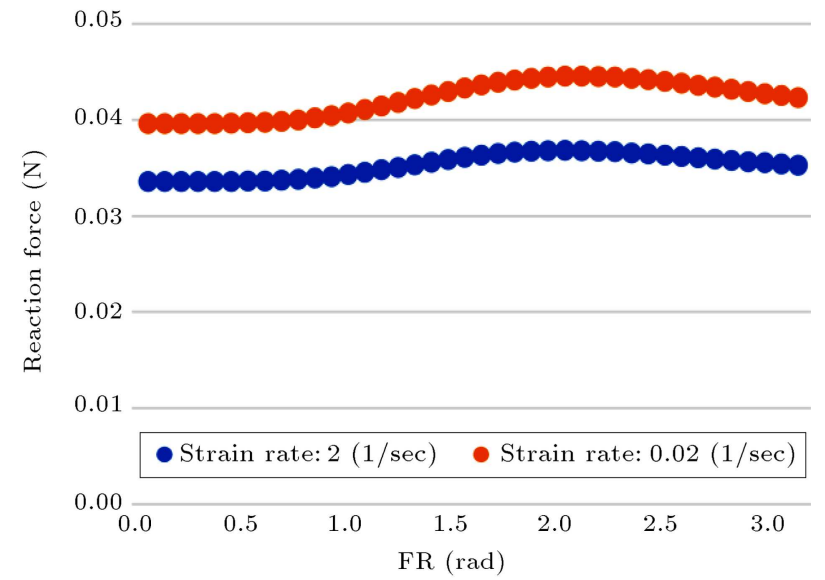

Figure 4. Indenter reaction force for all FRs and both strain rates; FR: fibrillar rotation.

To elaborate the effects of FR, some detailed contour plots were presented for a case with a strain rate of $2 \mathrm{Sec}^{-1}$ in order to compare the model without rotation with its counterpart with $2 \mathrm{Rad}$ rotation (Figure 5). The contour of the axial fibrillar stress implies that the stress resisted by the collagen fibers in the middle layer rose approximately by $210 \%$ of the initial value, demonstrating a more noticeable variation in stress. In contrast, the fluid pressure varied slightly in only SZ, revealing that FR mainly altered the load contributions of the AC solid parts. Finally, the Mises stress distribution signifies that FR could regionally affect the stress distribution. In all of the plots, a regional increase in the AC stress is observable, which might have a degenerative effect on the collagen network and can give rise to the development of osteoarthritis [11,29]. Although the rotations only affect the upper layer, it again supports the hypothesis that the AC FR might have significant mechanical effects and, consequently, could be considered as a new insight into the degenerative nature of knee rotational deformity $[15,17]$.

Although this study utilized some well-established and validated constitutive equations, some of the $\mathrm{AC}$ parameters were not incorporated to reduce the computational costs. First, the constitutive equations did not capture the viscosity of fibers and AC intrinsic swelling behavior $[6,18]$ which could have influenced the results. Nonetheless, they might not change the overall interpretation of the results substantially and have minor significance considering the aim of this study. Second, considering the extremely high value of the AC permeability, its fluid-dependent transient response outweighs the transient response governed by the viscosity of the fluid phase; subsequently, such viscosity was omitted. Third, this study aimed to provide a completely general explanation of FR to disclose their possible role in the mechanical response. Thus, the more exact patient-specific data, such as 


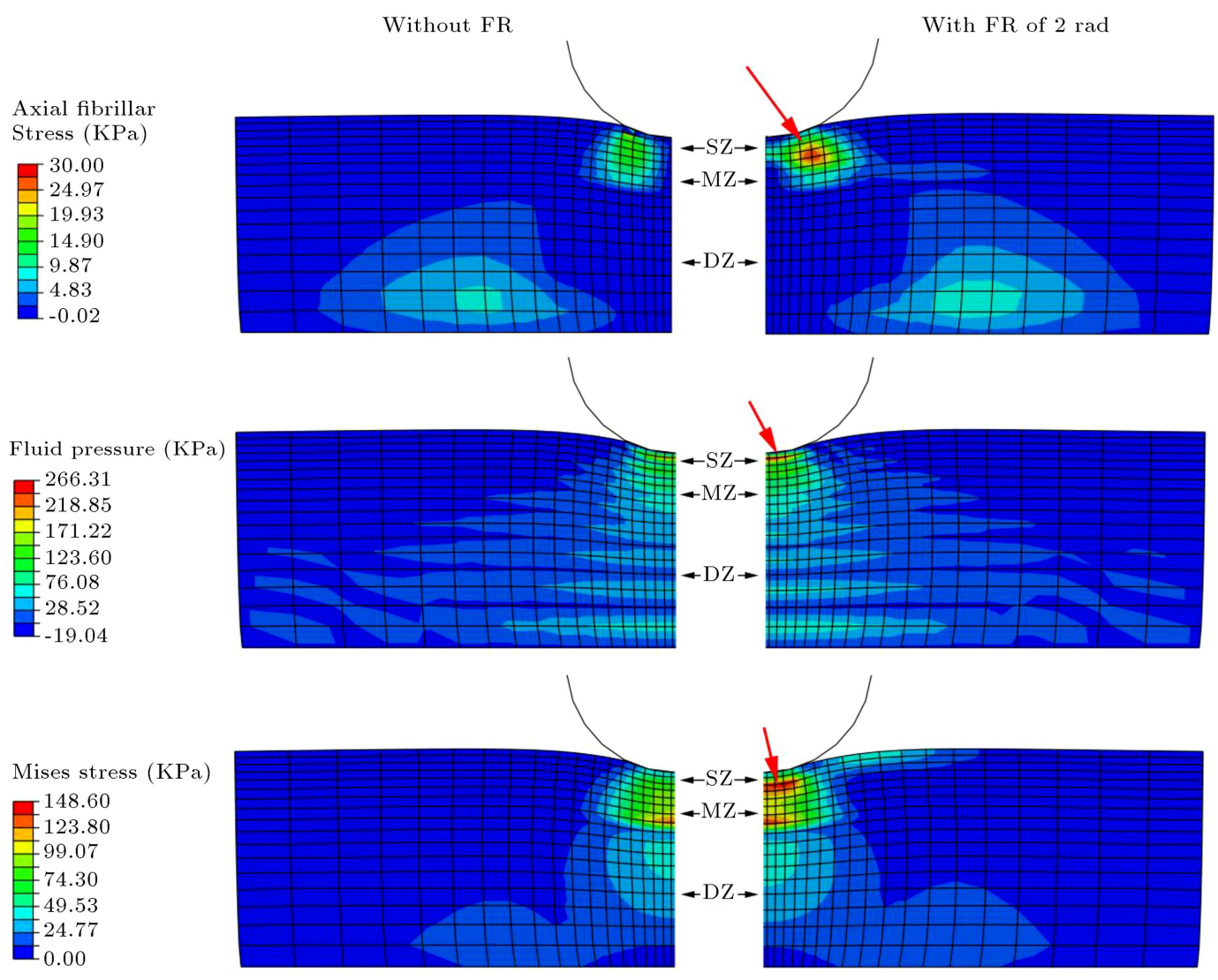

Figure 5. Plot contours of axial fibrillar stress, fluid (pore) pressure, and mises stress for 0 and 2 Rad FRs. Red arrows indicate the main affected regions. Note that the fibrils contributed merely to the stiffness of solid material and were not geometrically modeled. SZ: Superficial Zone; MZ: Middle Zone; DZ: Deep Zone; and FR: Fibrillar Rotation.

the exact image-based models of AC collagen network, were not captured, which could be addressed in future studies.

\section{Conclusions}

The present study presented promising results that could be used for simulating the possible rotations and arcade-like distributions of the fibrils simultaneously, if applicable. Accordingly, the excessive Fibrillar Rotation (FR) could regionally affect the Articular Cartilage (AC) mechanical response and increase the fibrillar stress contributions, which could have degenerative effects. Therefore, FR should be investigated in the future tissue-scale studies. However, since these rotations did not alter the elasticity of tissue, they are of insignificant importance in contact mechanical analysis of the knee joint scale AC studies.

\section{Acknowledgments}

The authors would like to thank Dr. Michael Stender (University of Colorado) for providing some precious computational information.

\section{Nomenclature}

Z

$\boldsymbol{\sigma}^{F}$

$\boldsymbol{\sigma}^{C O L}$

$\boldsymbol{\sigma}^{M A T} \quad$ Non-fibrillar Cauchy stress

$\boldsymbol{\sigma}^{\text {TOT }} \quad$ Total Cauchy stress

$n \quad$ Unit vector in the current direction of each fibril

$J \quad$ Determinant of the deformation gradient tensor

$\lambda \quad$ Elongation of each fibril

$\rho$ Relative volume fraction of the collagen fibrils in each direction

$E_{f}, M \quad$ Material parameters

$\phi^{0} \quad$ Initial solid volume fraction

$B \quad$ Left Cauchy-Green deformation tensor

$G_{m} \quad$ Shear modulus

$\theta \quad$ Rotation of fibrils 


\section{References}

1. Mow, V.C. and Huiskes, R., Basic Orthopaedic Biomechanics \& Mechano-Biology, Lippincott Williams \& Wilkins (2005).

2. Lories, R.J. and Luyten, F.P. "The bone-cartilage unit in osteoarthritis", Nat. Rev. Rheumatol., 7(1), pp. 4349, Jan. (2011).

3. Korhonen, R.K., Julkunen, P., Wilson, W., et al. "Importance of collagen orientation and depth-dependent fixed charge densities of cartilage on mechanical behavior of chondrocytes", J. Biomech. Eng., 130(2), p. 21003 (2008).

4. Taffetani, M., Griebel, M., Gastaldi, D., et al. "Poroviscoelastic finite element model including continuous fiber distribution for the simulation of nanoindentation tests on articular cartilage", J. Mech. Behav. Biomed. Mater., 32, pp. 17-30 (2014).

5. Wilson, W., van Donkelaar, C.C., van Rietbergen, B., et al. "Stresses in the local collagen network of articular cartilage: a poroviscoelastic fibril-reinforced finite element study", J. Biomech., 37(3), pp. 357-366 (2004).

6. Li, L.P. and Herzog, W. "The role of viscoelasticty of collagen fibers in articular cartilage: theory and numerical formulation", Biorheology, 41(3-4), pp. 181194 (2004).

7. Ateshian, G.A., Rajan. V., Nadeen, O., et al. "Modeling the matrix of articula cartilage using a continuous fiber angular distribution predicts many observed phenomena", J. Biomech. Eng., 131(6), pp. 1-34 (2009).

8. Stender, M.E., Raub, C.B., Yamauchi, K.A., et al. "Integrating qPLM and biomechanical test data with an anisotropic fiber distribution model and predictions of TGF- $\beta 1$ and IGF- 1 regulation of articular cartilage fiber modulus", Biomech. Model. Mechanobiol., 12(6), pp. 1073-1088 (2012).

9. Klets, O., Mononen, M.E., Tanska, P., et al. "Comparison of different material models of articular cartilage in 3D computational modeling of the knee: Data from the Osteoarthritis Initiative (OAI)", J. Biomech., 49(16), pp. 3891-3900 (2016).

10. Stender, M.E., Regueiro, R.A., and Ferguson, V.L. "A poroelastic finite element model of the bonecartilage unit to determine the effects of changes in permeability with osteoarthritis", Comput. Methods Biomech. Biomed. Engin., 20(3), pp. 319-331 (2017).

11. Stender, M.E., Carpenter, R.D., Regueiro, R.A., et al. "An evolutionary model of osteoarthritis including articular cartilage damage, and bone remodeling in a computational study", J. Biomech., 49(14), pp. 35023508 (2016).

12. Wilson, W., Huyghe, J.M., and Van Donkelaar, C.C. "Depth-dependent compressive equilibrium properties of articular cartilage explained by its composition", Biomech. Model. Mechanobiol., 6(1-2), pp. 43-53 (2007).
13. Mononen, M.E., Julkunen, P., Töyräs, J., et al. "Alterations in structure and properties of collagen network of osteoarthritic and repaired cartilage modify knee joint stresses", Biomech. Model. Mechanobiol., 10(3), pp. 357-369 (2011).

14. Wilson, W., van Donkelaar, C.C., van Rietbergen, B., et al. "A fibril-reinforced poroviscoelastic swelling model for articular cartilage", J. Biomech., 38(6), pp. 1195-1204 (2005).

15. Matsui, Y., Kadoya, Y., Uehara, K., et al. "Rotational deformity in varus osteoarthritis of the knee: Analysis with computed tomography", Clin. Orthop. Relat. Res., 433, pp. 147-151 (2005).

16. Shirazi, R., Shirazi-Adl, A., and Hurtig, M. "Role of cartilage collagen fibrils networks in knee joint biomechanics under compression", J. Biomech., 41(16), pp. 3340-3348 (2008).

17. Hosseini, S.M., Wu, Y., Ito, K., et al. "The importance of superficial collagen fibrils for the function of articular cartilage", Biomech. Model. Mechanobiol., 13(1), pp. 41-51 (2014).

18. Quiroga, J.M.P.P., Wilson, W., Ito, K., et al. "Relative contribution of articular cartilage's constitutive components to load support depending on strain rate", Biomech. Model. Mechanobiol., 16(1), pp. 151-158 (2017).

19. Meng, Q., An, S., Damion, R.A., et al. "The effect of collagen fibril orientation on the biphasic mechanics of articular cartilage", J. Mech. Behav. Biomed. Mater., 65, pp. 439-453 (2016).

20. Mow, V.C., Kuei, S.C., Lai, W.M., et al. "Biphasic creep and stress relaxation of articular cartilage in compression: Theory and experiments", J. Biomech. Eng., 102(1), p. 73 (1980).

21. Sajjadinia, S.S., Haghpanahi, M., and Razi, M. "Computational simulation of the multiphasic degeneration of the bone-cartilage unit during osteoarthritis via indentation and unconfined compression tests", Proc. Inst. Mech. Eng. Part H J. Eng. Med., 233(9), pp. 871-882 (2019).

22. Benninghoff, A. "Form und Bau der Gelenkknorpel in ihren Beziehungen zur Funktion", Z. Anat. Entwicklungsgesch., 76(1-3), pp. 43-63 (1925).

23. Li, L.P., Cheung, J.T.M., and Herzog, W. "Threedimensional fibril-reinforced finite element model of articular cartilage", Med. Biol. Eng. Comput., 47(6), pp. 607-615 (2009).

24. Gupta, S., Lin, J., Ashby, P., et al. "A fiber reinforced poroelastic model of nanoindentation of porcine costal cartilage: A combined experimental and finite element approach", J. Mech. Behav. Biomed. Mater., 2(4), pp. 326-338 (2009).

25. Lipshitz, H., Etheredge, R., and Glimcher, M.J. "In vitro wear of articular cartilage", J. Bone Joint Surg. Am., 57(4), pp. 527-534 (1975). 
26. Rieppo, J. "Poster: spatial determination of water, collagen and proteogycan content by fourier transform infrared imaging and digital densitometry", 50th Annu. Meet. Orthop. Res. Soc., no. Evo 5173, p. Poster No: 1021 (2004).

27. Shapiro, E.M., Borthakur, A., Kaufman, J.H., et al. "Water distribution patterns inside bovine articular cartilage as visualized by $1 \mathrm{H}$ magnetic resonance imaging", Osteoarthr. Cartil., 9(6), pp. 533-538 (2001).

28. Freutel, M., Schmidt, H., Dürselen, L., et al. "Finite element modeling of soft tissues: Material models, tissue interaction and challenges", Clin. Biomech., 29(4), pp. 363-372 (2014).

29. Mononen, M.E., Tanska, P., Isaksson, H., et al. "A novel method to simulate the progression of collagen degeneration of cartilage in the knee: Data from the osteoarthritis initiative", Sci. Rep., 6(1), p. 21415 (2016).

30. Below, S., Arnoczky, S.P., Dodds, J., et al. "The split-line pattern of the distal femur: A considera- tion in the orientation of autologous cartilage grafts", Arthroscopy, 18(6), pp. 613-617 (2002).

\section{Biographies}

Seyed Shayan Sajjadinia is currently a $\mathrm{PhD}$ student in Computer Science. He received his BSc and MSc in Mechanical and Biomedical Engineering, respectively. His current research interest is application of machine learning and numerical methods in advanced orthopedic simulations.

Mohammad Haghpanahi is currently a Full Professor at Iran University of Science and Technology. His research interests include orthopedic and sport biomechanics, stress analysis of systems through finite element analysis, static and fatigue analysis of welded joints, vibration and modal analysis of mechanical systems, and vocational education and its role in technology transfer. 